\title{
Homomorphism-homogeneous relational structures
}

\author{
Peter J. Cameron* \\ School of Mathematical Sciences \\ Queen Mary, University of London \\ Mile End Road, London E1 4NS, UK \\ $p \cdot j \cdot c a m e r o n @ q m u l \cdot a c \cdot u k$ \\ and \\ Jaroslav Nešetřril ${ }^{\dagger}$ \\ Department of Applied Mathematics and Institute of Theoretical Computer Sciences \\ Charles University \\ Malostranské Nám. 25, 11800 Praha, Czech Republic \\ nesetril@kam.mff.cuni.cz
}

\begin{abstract}
We study relational structures (especially graphs and posets) which satisfy the analogue of homogeneity but for homomorphisms rather than isomorphisms. The picture is rather different. Our main results are partial characterisations of countable graphs and posets with this property; an analogue of Fraïssé's Theorem; and representations of monoids as endomorphism monoids of such structures.
\end{abstract}

${ }^{*}$ This research was carried out while the first author was visiting KAM/ITI in Prague. He is grateful to the Department and Institute for their hospitality.

${ }^{\dagger}$ Supported by a Czech grant LN00A056 and ICREA, Barcelona. 


\section{Introduction}

A graph $G$ (or more general relational structure) is homogeneous if any isomorphism between finite induced subgraphs of $G$ can be extended to an automorphism of $G$. The homogeneous graphs can be recognised by the fact that their collections of finite subgraphs have the amalgamation property (Fraïssé's Theorem). The finite homogeneous graphs were determined by Gardiner [8] and the countably infinite ones by Lachlan and Woodrow [11]. Other determinations of homogeneous structures in various classes include posets (Schmerl [15]), tournaments (Lachlan [10]), permutations (Cameron [3]), and digraphs (Cherlin [4]). These structures are important in many parts of mathematics: see Hubička and Nešetřil $[13,9]$ for the connection with Ramsey theory, for example.

In this paper we consider what happens if we replace "isomorphism" in the definition of homogeneity by "homomorphism". (A homomorphism of a graph, for example, is a function which maps vertices to vertices and preserves the edges.)

There are several different conditions. We say that a graph $G$ belongs to the class

- HH, if every homomorphism from a finite subgraph of $G$ into $G$ extends to a homomorphism from $G$ to $G$;

- $\mathrm{MH}$, if every monomorphism from a finite subgraph of $G$ into $G$ extends to a homomorphism from $G$ to $G$;

- MM, if every monomorphism from a finite subgraph of $G$ into $G$ extends to a monomorphism from $G$ to $G$.

Clearly both $\mathrm{HH}$ and $\mathrm{MM}$ are included in the class $\mathrm{MH}$. So we begin with some structural results for MH graphs. Later we show that it is the class MM in which an analogue of Fraïssé's theory can be developed for arbitrary relational structures.

Proposition 1.1 (a) Any disjoint union of complete graphs all of the same size is $\mathrm{HH}$, and hence $\mathrm{MH}$. If a disjoint union of complete graphs is $\mathrm{MH}$, then the complete graphs all have the same size.

(b) If an MH graph is disconnected, or if it is finite, then it is a disjoint union of complete graphs of the same size. 
(c) If an MH graph is connected, then it has diameter at most 2 and every edge is contained in a triangle. Moreover, if $C$ is a finite maximal clique and $v$ any vertex, then $|C|$ is greater than the degree of $v$.

Proof The first part of (a) is clear. Moreover, if an MH graph has two components $A$ and $B$ which are complete, then for $a \in A$ and $b \in B$, the map $a \mapsto b$ extends to a homomorphism which maps $A$ injectively to $B$, so $|A| \leq|B|$. Similarly $|B| \leq|A|$. So $|A|=|B|$ by the Cantor-Schröder-Bernstein theorem.

Suppose that $G$ is $\mathrm{MH}$ and has a component which is not complete: equivalently, it has an induced path $x, y, z$. For any two distinct vertices $a, b$, the map $x \mapsto a, z \mapsto b$ extends to a homomorphism, which maps $z$ to a common neighbour of $a$ and $b$. This shows that $G$ is connected and has diameter 2 and every edge is in a triangle. This proves (b) for disconnected graphs, and also the first part of (c).

Let $C$ be a finite clique in an MH graph and suppose there is a vertex $v$ with degree at least $|C|$. Any injective map from $|C|$ neighbours of $v$ to $C$ extends to a homomorphism, which must map $v$ to a common neighbour of $C$, so $C$ is not maximal.

Now suppose that $G$ is finite $\mathrm{MH}$, and let it be a minimal counterexample to the statement that it is a disjoint union of complete graphs of the same size. Then $G$ is connected. For any vertex $v$, the set of neighbours of $v$ is $\mathrm{MH}$, and so by assumption is a disjoint union of, say, $l$ complete graphs of size $k$. Then $G$ has a maximal clique of size $k+1$, while $v$ has degree $k l$. So we have $k+1>k l$, whence $k \geq k l$, and $l=1$. Then the set of neighbours of $v$ is a complete graph. Taking $v$ to be a vertex of maximum degree in its component, we see that this component is a complete graph of size $k+1$.

The classification of finite MM graphs is simpler:

Proposition 1.2 The only finite MM graphs are the complete and null graphs.

Proof Suppose that $G$ is a finite MM graph which is neither complete nor null. Let the vertices $a$ and $b$ be adjacent, and the vertices $c$ and $d$ be non-adjacent. Then the map $c \mapsto a, d \mapsto b$ extends to a monomorphism of $G$, which strictly increases the number of edges, which is clearly impossible.

\section{Graphs spanned by $R$}

Let $R$ be the countable random graph (the "Rado graph", [14]). Recall that $R$ is characterised as a countable graph with the property that, if $U$ and $V$ are finite 
disjoint sets of vertices, there is a vertex $z$ joined to all vertices in $U$ and to none in $V$. See [2] for more information about $R$.

Proposition 2.1 (a) A countable graph contains $R$ as a spanning subgraph if and only if it has the property that any finite set of vertices has a common neighbour.

(b) Any graph containing $R$ as a spanning subgraph is $H H$ and $M M$, and hence MH.

(c) If $G$ is an $M H$-graph which does not contain $R$ as a spanning subgraph, then there is a bound on the size of claws (induced stars $K_{1, n}$ ) in $G$.

Proof (a) The property holds in $R$, and hence certainly in any graph obtained by adding extra edges.

Conversely, let $G$ be a countable graph satisfying the property. Construct a bijection between $R$ and $G$ by the back-and-forth method, except that in going from $R$ to $G$ we don't insist that non-edges are preserved. In more detail: we define a map $f: R \rightarrow G$ recursively. At odd-numbered steps, take the first vertex of $R$ on which $f$ is not yet defined, and map it to a common neighbour of the range of $f$. At even-numbered stages, take the first vertex $v$ not in the range of $f$, choose $v^{\prime} \in R$ such that, for all $u$ in the domain of $f, u \sim v^{\prime}$ if and only if $f(u) \sim v$.

(b) If the property of (a) holds, then certainly homomorphisms extend: if we have defined $f$ on $v_{0}, \ldots, v_{n-1}$, then choose $f\left(v_{n}\right)$ to be any vertex adjacent to all of $f\left(v_{0}\right), \ldots, f\left(v_{n-1}\right)$. Moreover, if $f$ is one-to-one, then so is the extension.

(c) Suppose that $G$ is a countable $\mathrm{MH}$ graph which contains claws of unbounded size. Let $U$ be a finite set of vertices, with $|U|=n$. Find a claw $K_{1, n}$ in $G$ and map its independent vertices bijectively to $U$. The remaining vertex is mapped to a neighbour of $U$. So $G$ satisfies the condition of (a).

\section{Corollary 2.2}

(a) There is a countable graph which is homomorphism-homogeneous but is automorphismrigid.

(b) There is a countable graph which is homomorphism-homogeneous but its complement is homomorphism rigid.

Proof The graph 


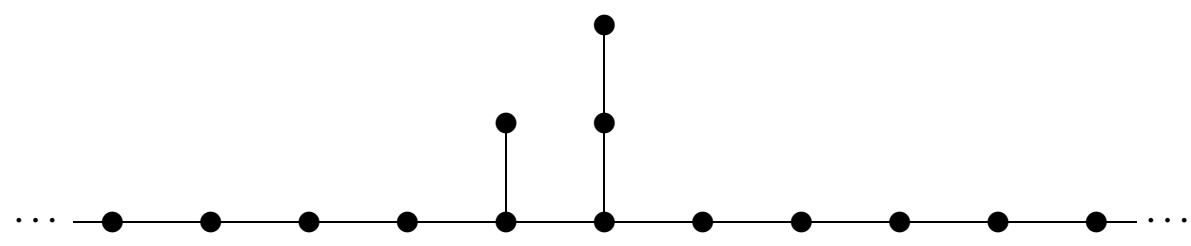

is automorphism-rigid and for every finite subset $U$ there is a vertex joined to no vertex in $U$. So the complement is also automorphism-rigid and contains $R$ as a spanning subgraph. This proves (a).

To prove (b) it suffices to prove that there exists a countable rigid graph with all its degrees $\leq 3$. The complement the contains $R$ as a spanning subgraph.

Here are two questions which we have not been able to resolve.

Problem 1 Is there a graph which is $\mathrm{MH}$ but not $\mathrm{HH}$ ?

We remark that for more general structures than graphs we prove below that the classes $\mathrm{MH}$ and $\mathrm{HH}$ different.

Problem 2 Is there a countable graph which is $\mathrm{HH}$ but not a disjoint union of complete graphs and does not contain $R$ as a spanning subgraph?

A positive answer to this problem would yield a graph which contains a finite set of vertices with no common neighbours, and there is a bound on the size of its claws. One famous class of graphs with bounded claw size consists of line graphs $L(G)$ of graphs; these contain no 3-claw $K_{1,3}$. We show that at least in this class we obtain no new examples.

Proposition 2.3 Let $G$ be a finite or countable graph with the propery that $L(G)$ is $M H$. Then $G$ is a disjoint union of stars of the same size (and hence $L(G)$ is a disjoint union of complete graphs of the same size).

Proof By Propositions 1.1 and 1.2, we can assume that $G$ is infinite and connected with bounded diameter. So $G$ contains a vertex $v$ of infinite degree.

First we show that $G$ is triangle-free. Suppose that $\{a, b, c\}$ is a triangle in $G$, and let $p, q, r, s$ be neighbours of $v$. The map $v p \mapsto a b, v q \mapsto b c, v r \mapsto c a$ of $L(G)$ extends to a homomorphism of $L(G)$, under which $v s$ must map to an edge meeting all three edges of the triangle, which is impossible.

Now we show that any neighbour of $v$ has degree 1 . For suppose that $p$ is a neighbour of $v$ which is also adjacent to a vertex $x$ (necessarily not adjacent to $v$ ), and let $q$ and $r$ be two further neighbours of $v$. The map $v q \mapsto v q, v r \mapsto v p$, 
$p x \mapsto p x$ extends to a homomorphism, which must map $v p$ to an edge containing $v$ and meeting $p x$; this edge cannot be $v p$, and if it is $v x$ then the graph contains a triangle. So no such vertex can exist.

Thus, the connected component containing $v$ is an infinite star, and we are done.

More generally, let $\mathcal{K}(k, l)$ be the class of finite graphs defined as follows, where $k$ and $l$ are integers with $1<l<k$ : the vertex set is an arbitrary set $\mathcal{M}$ of $k$-sets; two vertices are adjacent if and only if they intersect in at least $l$ points. We call this the $(\geq l)$-intersection graph of $\mathcal{M}$. (So line graphs form the class $\mathcal{K}(2,1)$. More generally, in the case $l=1$, we refer to such a graph as the intersection graph of the collection of $k$-sets.)

Now if the $k$-sets $\left(X, Y_{1}, \ldots, Y_{r}\right)$ form a claw in the $(\geq l)$-intersection graph, then the intersections $X \cap Y_{i}$ for $1 \leq i \leq r$ all have size at least $l$ and are all distinct; so certainly there is no $2^{k}$-claw in such a graph. We would like to prove that no countable graph of this type can be homogeneous unless it is a disjoint union of complete graphs. Here is a first step.

Proposition 2.4 For $k \geq 3$, let $\left(\begin{array}{l}X \\ k\end{array}\right)$ denote the set of all $k$-subsets of a countable set. Then the $(\geq l)$-intersection graph of $\left(\begin{array}{l}X \\ k\end{array}\right)$ is not $M H$.

Proof There always exists a ( $\geq l$ )-intersecting family of $k$-subsets with the property that no further $k$-set intersects every set in the family. For example, the set $\left(\begin{array}{l}Y \\ k\end{array}\right)$ of all $k$-subsets of a $(2 k-l)$-set $Y$ obviously has this property, since a set containing fewer than $k$ points of $Y$ has intersection smaller than $l$ with some set in $\left(\begin{array}{l}Y \\ k\end{array}\right)$.

Now there is also an infinite star (a set of $k$-sets containing $l$ common points). Now the map taking $\left(\begin{array}{c}2 k-l \\ k\end{array}\right)$ sets of the star bijectively to $\left(\begin{array}{l}Y \\ k\end{array}\right)$ cannot be extended to a further set of the star.

A final observation about MM graphs:

Proposition 2.5 Any infinite non-null MM graph contains an infinite complete subgraph.

Proof Let $G$ be such a graph.

Suppose first that $G$ is disconnected, and so a union of complete graphs. If the components are finite (of size $n>1$, say), take two points $a, b$ in different 
components and map them to two points in the same component; it is clear that no monomorphism can extend this map. So the components are infinite, as required.

Suppose that $G$ does not contain $R$, so that it fails to contain the star $K_{1, n}$ for some $n$. Since $G$ has diameter 2, some vertex has infinite degree, and its neighbourhood has no null graph of size $n$. By Ramsey's Theorem it contains an infinite complete graph.

Finally, if $G$ contains $R$, the result is clear.

\section{Posets}

A homomorphism of posets is a map $f$ such that, if $x<y$, then $f(x)<f(y)$. Now the definitions of the classes $\mathrm{HH}, \mathrm{MH}$ and $\mathrm{MM}$ of posets are exactly as for graphs. Here is the start of an attempt to classify the MH posets.

First, the analogue of the fact that graphs containing $R$ as spanning subgraph have the MM and HH properties also hold here. Let $U$ denote the generic (universal and homogeneous) countable poset. An extension of $U$ is a poset $P$ on the same set such that, if $x \leq_{U} y$, then $x \leq_{P} y$.

Proposition 3.1 (a) A countable poset $P$ is an extension of $U$ if and only if it has the following property $(\dagger)$ : for any two finite sets $A$ and $B$ with $A<B$, there is a point $z$ such that $A<z<B$.

(b) Any extension of $U$ has the HH and MM properties.

Proof (a) The argument is similar to that for graphs. Recall that $U$ is characterised by the property that, for any finite disjoint sets $A, B, C$ with $A<B$ and, for all $a \in A, b \in B, c \in C$, we have $c \nless a$ and $b \nless c$, there is a point $z$ with $A<z<B$ and $z$ and $c$ incomparable for all $c \in C$.

Now construct a bijection from $U$ to $P$ which preserves comparability as follows. Enumerate $P$ and $U$. Suppose that $f$ has been defined on $u_{1}, \ldots, u_{n} \in U$. If $n$ is even, choose the first unused point $x$ of $U$; let $A$ and $B$ be the subsets of $\left\{u_{1}, \ldots, u_{n}\right\}$ consisting of points less than and greater than $x$ respectively. Then $A<B$, so $f(A)<f(B)$; by $(\dagger)$, there is a point $z$ such that $A<z<B$, and we can map $x$ to $z$. If $n$ is odd, choose the first unused point $y$ of $P$, and choose a point $z \in P$ incomparable with all of $u_{1}, \ldots, u_{n}$; then map $z$ to $y$.

(b) Let $P$ be an extension of $U$, and $f$ a homomorphism between finite subsets of $P$. Now we can extend $f$ to all of $P$ as follows. Suppose that $f$ has been defined on $p_{1}, \ldots, p_{n}$. Let $A$ and $B$ be the sets of elements of $\left\{p_{1}, \ldots, p_{n}\right\}$ which are 
respectively less than and greater than $p_{n+1}$. As before, choose $z$ with $f(A)<z<$ $f(B)$, and map $p_{n+1}$ to $z$. The extension is one-to-one everywhere except possibly on the points where $f$ was initially defined.

Now we gather a few facts about $\mathrm{MH}$ posets. Clearly any antichain is $\mathrm{HH}$ and MM.

Proposition 3.2 Let $P$ be a countable poset which is MH but not an antichain.

(a) Any maximal chain in $P$ is dense and without endpoints.

(b) If $P$ is disconnected then it is a disjoint union of incomparable chains each isomorphic to the rationals.

(c) If there is a 2-element antichain in $P$ which has an upper bound, then any finite antichain has an upper bound.

Proof (a) By assumption, there exist $a$ and $b$ with $a<b$. Extending the map $a \mapsto x$ for any $x$, we see that there is an element above $x$. (Dually there is an element below $x$.) In particular, there is a 3-element chain $a<b<c$. Now, if $x<y$, extending the map $a \mapsto x, c \mapsto y$, we see that the image of $b$ is an element $z$ with $x<z<y$.

(b) If $P$ has a component which is not a chain, then it contains a 2-element antichain with either an upper or a lower bound. By the MH property, every 2element antichain has a bound; so there is only one connected component.

(c) As in (b), if some 2-element antichain has an upper bound, then so does every 2-element antichain. Now, inductively, let $a_{1}, \ldots, a_{n}$ be an antichain. Let $b_{1}$ be an upper bound of $a_{i}$ and $a_{i+1}$ for $i=1, \ldots, n-1$. Now the set of maximal elements among $b_{1}, \ldots, b_{n-1}$ is an antichain of size smaller than $n$; by induction it has an upper bound $c$, which is also an upper bound for $a_{1}, \ldots, a_{n}$.

It follows that all homogeneous posets have the MM property. A bit surprisingly there is another example (which is also not an extension of the generic poset $U$, but is more analogous to a claw-free graph). Recall that a tree order is a poset in which the elements below any given element $x$ form a chain). Tree orders may have large symmetry groups (see Droste [5]) while not being homogeneous (in the usual sense). We prove that they may have the MM property. Let $T$ be a countable tree order satisfying the following additional properties:

(a) $T$ is dense; 
(b) $T$ has neither maximal nor minimal element;

(c) No finite subset of $T$ has a proper infimum (i.e. infima and minimal elements of finite subsets coincide);

(d) $T$ is infinitely branching (i.e. i.e. for every $x<y$ there exists an antichain $z_{i} ; i=1,2, \ldots$, with $x<z_{i}$ and $y \not z z_{i}$ for every $\left.i=1,2, \ldots\right)$.

Extending Cantor's characterization of countably dense linear orders one can see that $T$ is up to an isomorphism uniquely determined by the above properties. It can be obtained as follows: First we split the set of rational numbers in two dense sets, say $D, D^{\prime}$. We form an infinite tree of copies of $D$ by adding infinitely many branching copies of $D$ at any element of $D^{\prime}$ and continuing recursively in this way.

We have the following:

Proposition 3.3 (a) $T$ is not homogeneous (in the usual sense);

(b) T has the MM property.

Proof The reason why $T$ is not homogeneous in the ordinary sense can be seen from this picture.

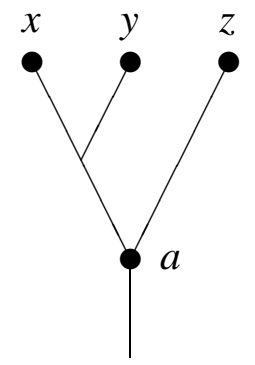

It is clear that no automorphism can interchange $x$ with $z$ and fix $y$. But there is a monoomorphism that will do this: simply map the points below $x, y, z$ to suitable points below $a$.

To prove (b), it suffices to prove that for any $x \notin A$ any monomorphism $f$ : $A \longrightarrow B$ may be extended to a monomorphism $f^{\prime}: A^{\prime} \longrightarrow B^{\prime}$ where $A^{\prime}=A \cup\{x\}$. This may be seen as an extension of the above example: Given $x \notin A$ denote by $A_{x}$ the set of all $z \in A$ such that $x \leq z$. Let $B_{x}=\left\{f(y) ; y \in A_{x}\right\}$. Consider the infimum $a$ of the set $A_{x} \cup B_{x}$ (it may not belong to $T$ ). If $a \geq x$ then we put $f^{\prime}(x)=x$. If $a<x$ then necessarily $B_{x} \backslash A_{x}$ is non-empty. In this situation we distinguish two cases: 
- If there is an element $z$ of $A$ satisfying $a<z<x$, we choose maximal such $z$, and the we let $f^{\prime}(x)$ be the element above $f(z)$ close enough to be distinct from all of $A$ and $B$.

- If there is no element of $A$ satisfying $a<z<x$ we let $f^{\prime}(x)$ be an element bellow $a$ which such that $f^{\prime}(x)>z$ for all $z<a, z \in A \cup B$.

\section{Homogeneity and amalgamation}

The definitions of the classes $\mathrm{HH}, \mathrm{MH}$ and MM work in the same way for arbitrary relational structures as for graphs or posets. We are going to develop a characterisation of MM structures in general. As usual, the age of a structure is the class of finite structures embeddable in it; and the joint embedding property or JEP is as usual: $C$ has the JEP if any two members of $C$ can be embedded in some member of $\mathcal{C}$.

The mono-amalgamation property (for short, MAP) of a class $C$ of finite relational structures is the following assertion:

For any $A, B_{1}, B_{2} \in \mathcal{C}$, and any maps $f_{i}: A \rightarrow B_{i}$ (for $i=1,2$ ) such that $f_{1}$ is an embedding (an isomorphism to an induced substructure) and $f_{2}$ a monomorphism, there exists $C \in C$ and monomorphisms $g_{i}$ : $B_{i} \rightarrow C$ for $i=1,2$ such that $g_{1} \circ f_{1}=g_{2} \circ f_{2}$ and $g_{2}$ is an embedding.

Note the asymmetry between $B_{1}$ and $B_{2}$ !

The mono-extension property of a structure $M$ with age $C$ is the following property:

If $B \in C$ and $A$ is an induced substructure of $B$, then every monomorphism $A \rightarrow M$ extends to a monomorphism $B \rightarrow M$.

Now our analogue of Fraïssé's Theorem is the following result.

Proposition 4.1 (a) A countable structure is MM if and only if it has the monoextension property.

(b) The age of any MM-structure has the mono-amalgamation property.

(c) If a class $\mathcal{C}$ of finite relational structures is isomorphism-closed, closed under induced substructures, has only a countable number of isomorphism classes, and has the JEP and the MAP, then there is a countable MM structure $M$ whose age is equal to $C$. 
Proof (a) If $M$ has the mono-extension property, then clearly any monomorphism from a finite substructure of $M$ can be extended (one point at a time) to a monomorphism of $M$.

Conversely, suppose that $M$ is an MM structure. Let $B$ be a structure in the age of $M$ and $A \subseteq B$ : without loss of generality, $B \subseteq M$. Suppose that $f$ is any monomorphism from $A$ into $M$. Then $f$ extends to a monomorphism $g$ of $M$, whose restriction to $B$ is the required monomorphism $B \rightarrow M$.

(b) Suppose that $M$ is an MM structure and let $A, B_{1}, B_{2}, f_{1}, f_{2}$ be as in the hypothesis of the mono-amalgamation property, with $A, B_{1}, B_{2}$ in the age of $M$. As in (a), we can assume that $B_{1}, B_{2} \subseteq M$ and $f_{1}$ is the identity on $A$. Now extend $f_{1}$ to a monomorphism $g$ of $M$; let $C=B_{1} g, g_{1}$ the restriction of this monomorphism to $B_{1}$, and $g_{2}$ the identity on $B_{2}$.

(c) We build the structure in stages; even and odd numbered stages achieve different parts of the construction. Suppose that $M_{i}$ has been constructed at stage $i$.

If $i$ is even, we can use the JEP to find a structure $M_{i+1}$ containing $M_{i}$ and any given structure $A \in C$.

If $i$ is odd, we select a pair $(A, B)$ of structures in $C$ with $A \subseteq B$. Now a given monomorphism $A \rightarrow M$ can be extended to a monomorphism $B \rightarrow M^{\prime}$ for some $M^{\prime} \supseteq M$, by the MAP. Applying this successively for each monomorphism $A \rightarrow$ $M_{i}$, we obtain the structure $M_{i+1}$ so that every monomorphism $A \rightarrow M_{i}$ extends to a monomorphism $B \rightarrow M_{i+1}$.

Arranging the stages so that every structure in $C$ occurs at some even stage and every pair $(A, B)$ at infinitely many odd stages, we finally build a countable structure whose age is $C$ and which has the mono-extension property, and hence is MM.

We say that a class $C$ having the properties of part (c) of the theorem is a mono-Fraïsé class, and that an MM structure with age $\mathcal{C}$ is a mono-limit of $\mathcal{C}$. Unlike the usual form of Fraïssé's Theorem, it is not the case that a class satisfying the mono-amalgamation property has a unique mono-limit (up to isomorphism). Indeed, there are many examples of graphs containing $R$ as spanning subgraph whose age is the class of all finite graphs.

However, any two such structures $M$ and $M^{\prime}$ must bear a certain resemblance to each other. For example, there is a monomorphism from $M$ to $M^{\prime}$ and vice versa. In fact, more is true; we can characterise this equivalence as follows.

Let $M$ and $M^{\prime}$ be two structures. We say that $M$ and $M^{\prime}$ are mono-equivalent if

- $\operatorname{Age}(M)=\operatorname{Age}\left(M^{\prime}\right)$; 
- every embedding of a finite substructure $A$ of $M$ into $M^{\prime}$ extends to a monomorphism from $M$ to $M^{\prime}$, and vice versa (with $M$ and $M^{\prime}$ reversed).

This turns out to be the relation which replaces isomorphism in our version of Fraïssé's Theorem:

Proposition 4.2 (a) Suppose that $M$ and $M^{\prime}$ are mono-equivalent structures. If $M$ is a MM structure, then $M^{\prime}$ is an MM structure too.

(b) Conversely, if $M$ and $M^{\prime}$ are $M M$ structures with $\operatorname{Age}(M)=\operatorname{Age}\left(M^{\prime}\right)$, then they are mono-equivalent.

Proof (a) Suppose that $M$ and $M^{\prime}$ are equivalent and that $M$ has the MM property. Take $A, B \in \operatorname{Age}\left(M^{\prime}\right)$ with $A \subseteq B$, and let $f: A \rightarrow M^{\prime}$ be a monomorphism. By assumption, we may assume that $B \subseteq M$.

Let $A^{\prime}$ be the image of $f$. Since $\operatorname{Age}(M)=\operatorname{Age}\left(M^{\prime}\right)$, we can find a copy $A^{\prime \prime}$ of $A^{\prime}$ within $M$; in other words, there is a monomorphism $\phi: A \rightarrow A^{\prime \prime}$ and an isomorphism $g: A^{\prime \prime} \rightarrow A^{\prime}$ such that $g \circ \phi=f$. Since $M$ has the MM property, the monomorphism $\phi$ extends to a monomorphism $\phi^{*}: M \rightarrow M$. Let $B^{\prime \prime}=\phi^{*}(B)$. Also, by assumption, the isomorphism $g$ extends to a monomorphism $g^{*}: M \rightarrow M^{\prime}$. Now the restriction of $g^{*} \circ \phi^{*}$ to $B$ is a monomorphism $B \rightarrow M^{\prime}$ extending the given monomorphism $f$. So $M^{\prime}$ has the mono-extension property, and hence it is an MM structure.

(b) Suppose that $M$ and $M^{\prime}$ are MM structures with the same age. Let $A$ be a finite substructure of $M$ and $f: A \rightarrow M^{\prime}$ an embedding. For any $B \supseteq A$, the monoextension property in $M^{\prime}$ allows us to extend $f$ to a monomorphism $B \rightarrow M^{\prime}$. So there is a monomorphism $M \rightarrow M^{\prime}$ extending $f$. Thus $M$ and $M^{\prime}$ are equivalent.

The above proof that the MM property for $M$ implies that of $M^{\prime}$ uses only half of the definition of equivalence. Let us say that $M \preceq M^{\prime}$ holds if

- $\operatorname{Age}(M) \supseteq \operatorname{Age}\left(M^{\prime}\right)$, and

- any embedding of a finite substructure of $A$ into $M^{\prime}$ extends to a monomorphism from $M$ to $M^{\prime}$.

This relation between structures defines a partial order on the set of equivalence classes. (The reverse ordering of ages looks strange, but consider graphs containing $R$ as spanning subgraph: intuitively, the more extra edges we add, the smaller 
the age becomes.) Then the proof of Proposition 4.2(a) actually shows that, if $M$ is an MM structure and $M \preceq M^{\prime}$, then also $M^{\prime}$ is an MM structure.

We mention one fact about this order for graphs.

Proposition 4.3 Let $R$ be the Rado graph. Then a countable graph $G$ satisfies $R \preceq G$ if and only if $R$ is a spanning subgraph of $G$.

Proof Since $R$ is universal, the condition $\operatorname{Age}(R) \supseteq \operatorname{Age}(G)$ is trivial.

Suppose that $R$ is a spanning subgraph of $G$, and let $f: A \rightarrow G$ be an embedding of a finite subgraph $A$ of $R$ into $G$. Since there is a common neighbour of $f(A)$ in $G$ by Proposition 2.1, it is always possible to extend $f$ to a monomorphism on one extra point.

Conversely, suppose that $R \preceq G$, and let $U$ be a finite set of vertices in $G$. Let $A$ be a subgraph of $R$ isomorphic to the subgraph $U$ (by means of the isomorphism $f$ ), and $z$ a common neighbour of $A$ in $R$. The map $f$ extends to a monomorphism from $R$ to $G$ (by assumption), and the image of $z$ is a common neighbour of $U$. So $G$ contains $R$ as a spanning subgraph, again by Proposition 2.1.

On the other hand, it is not true that if $M$ and $M^{\prime}$ are MM structures and $\operatorname{Age}(M) \supseteq \operatorname{Age}\left(M^{\prime}\right)$, then $M \preceq M^{\prime}$. For example, let $M$ be the Rado graph $R$, and $M^{\prime}$ the disjoint union of two infinite complete graphs. The map taking a non-edge in $M$ to a non-edge in $M^{\prime}$ clearly cannot be extended.

\section{Algebraic classes}

We say that a class $\mathcal{K}$ of finite structures has the ME-property if the classes of monomorphisms and embeddings coincide for $\mathcal{K}$.

Proposition 5.1 Let $\mathcal{K}$ be a mono-Fraïssé class of finite structures with the MEproperty. Then any two mono-limits of $\mathcal{K}$ are isomorphic.

Examples:

- all algebraic classes (containing only function and constant symbols)

- tournaments and directed graphs (see below). 
Commentary Clearly any monomorphism between finite algebras is an embedding. For if $\rho$ is an $r$-ary operation in the algebra and $f$ a monomorphism, then clearly $\rho\left(f\left(x_{1}\right), \ldots, f\left(x_{r}\right)\right)=f\left(\rho\left(x_{1}, \ldots, x_{r}\right)\right)$.

Now, given any loopless directed graph $D$, it is possible to define an algebra on the vertex set of $D$ with a single binary operation $\cdot$ by the rules

$$
\begin{aligned}
x \cdot x & =x, \\
\text { and if } x \neq y, \text { then } x \cdot y & = \begin{cases}x & \text { if there is an } \operatorname{arc} x \rightarrow y, \\
y & \text { otherwise }\end{cases}
\end{aligned}
$$

Any monomorphism of this algebra is a digraph embedding. Note that not all digraph monomorphisms are algebra monomorphisms! If the digraph is a tournament then the two types of monomorphisms coincide: see [12].

This example shows that, even in the case of algebras of a given type, we may have $2^{\aleph_{0}}$ non-isomorphic MM-structures (in this case, the algebras of the homogeneous digraphs determined by Cherlin [4]).

A more familiar example of this phenomenon occurs with abelian groups. For any set $\Pi$ of prime numbers, the direct product of the countable abelian group of exponent $p$ for all $p \in \Pi$ is homogeneous.

The above example of tournament algebras (sometimes called quasitrivial algebras) yield also an example of structures showing $\mathrm{MH} \neq \mathrm{HH}$ : Consider the algebra corresponding to the universal homogeneous tournament. This algebra is obviously an MM structure. However it fails to be an $\mathrm{HH}$ structure as every finite tournament may be extended to a finite tournament which is simple (i.e. does not have any non-trivial congruence), see $[6,12]$.

\section{Representing closed monoids}

There is a natural topology on $X^{X}$, for a countable set $X$, namely the product topology induced from the discrete topology on $X$. Thus the basic open sets are of the form

$$
\left\{f \in X^{X}: f\left(x_{i}\right)=y_{i} \text { for } i=1, \ldots, n\right\},
$$

where $x_{1}, \ldots, x_{n}, y_{1}, \ldots, y_{n} \in X$ and $x_{1}, \ldots, x_{n}$ are distinct. It is known that, in the induced topology on the symmetric group $\operatorname{Sym}(X)$, a permutation group $G$ is closed if and only if it is the automorphism group of a homogeneous relational structure on $X$ (see [1]). A similar observation holds here: 
Proposition 6.1 (a) A submonoid $S$ of $X^{X}$ is closed in the product topology on $X^{X}$ if and only if $S$ is the monoid $\operatorname{End}(M)$ of endomorphisms of a HH relational structure $M$ on $X$.

(b) A submonoid $S$ of the monoid of one-to-one maps $X \rightarrow X$ is closed in the product topology if and only if $S$ is the monoid of monomorphisms of a MM relational structure $M$ on $X$.

Proof (a) For each $n$, and each $\bar{x} \in X^{n}$, we take an $n$-ary relation $R_{\bar{x}}$ defined by

$$
R_{\bar{x}}(\bar{y}) \Leftrightarrow(\exists s \in S)(\bar{y}=s(\bar{x}))
$$

Let $M$ be the relational structure with relations $R_{\bar{x}}$ for all $n$-tuples $\bar{x}$ (and all $n$ ). We claim that $S$ acts as endomorphisms of $M$, that $M$ is $\mathrm{HH}$, and $\operatorname{End}(M)=S$.

For the first point, take $s \in S$ and $\bar{y} \in X^{n}$ such that $R_{\bar{x}}(\bar{y})$ holds; we must show that $R_{\bar{x}}(s(\bar{y}))$ holds. But $\bar{y}=s^{\prime}(\bar{x})$ for some $s^{\prime} \in S$; then $s(\bar{y})=s s^{\prime}(\bar{x})$, so the assertion is true.

Next, let $f$ be a homomorphism between finite subsets of $X$, say $f\left(x_{i}\right)=y_{i}$ for $i=1, \ldots, n$. Let $\bar{x}=\left(x_{1}, \ldots, x_{n}\right)$ and $\bar{y}=\left(y_{1}, \ldots, y_{n}\right)$. Now $S$ is a monoid and so contains the identity mapping. Thus, by definition, $R_{\bar{x}}(\bar{x})$ holds. Since $f$ is a homomorphism, $R_{\bar{x}}(\bar{y})$ holds. So by definition, there exists $s \in S$ such that $s(\bar{x})=\bar{y}$. Now $s$ is an endomorphism of $M$ extending $f$. So $M$ is HH.

Finally, to show that $\operatorname{End}(M)=S$, we know already that $S \subseteq \operatorname{End}(M)$ and have to prove the reverse inclusion. We must take $h \in \operatorname{End}(M)$ and show that every basic neighbourhood of $h$ contains an element of $S$, so that $h$ is a limit point of $S$. Since $S$ is assumed closed, we conclude that $h \in S$. Now each $n$-tuple $\bar{x}$ defines a basic neighbourhood of $h$, consisting of all functions $g$ such that $g(\bar{x})=h(\bar{x})$. Now $R_{\bar{x}}(\bar{x})$ holds; since $h$ is a homomorphism, $R_{\bar{x}}(h(\bar{x}))$ also holds, and by definition of $R_{\bar{x}}$ this means that there exists $s \in S$ with $h(\bar{x})=s(\bar{x})$, as required.

(b) The proof of this is entirely analogous, replacing homomorphisms by monomorphisms.

The relational structures constructed in the proof have infinitely relations of each arity. It would be interesting to recognise the monoids which are the endomorphism monoids (or monomorpism monoids) of homogeneous structures with only finitely many relations of each arity (these would be the analogue of the closed oligomorphic permutation groups, [1]), or even those with only finitely many relations altogether. 


\section{References}

[1] P. J. Cameron, Oligomorphic Permutation Groups, London Math. Soc. Lecture Notes 152, Cambridge University Press, Cambridge, 1990.

[2] P. J. Cameron, The random graph, pp. 331-351 in The Mathematics of Paul Erdős (J. Nešetřil and R. L. Graham, eds.), Springer, Berlin, 1996.

[3] P. J. Cameron, Homogeneous permutations, Electronic J. Combinatorics 9(2) (2002), \#R2 (9pp.)

[4] G. L. Cherlin, The classification of countable homogeneous directed graphs and countable homogeneous $n$-tournaments, Memoirs Amer. math. Soc. 621, American Math. Soc., Providence, RI, 1998.

[5] M. Droste, Structure of partially ordered sets with transitive automorphism groups, Memoirs Amer. Math. Soc. 57, American Math. Soc., Providence, RI, 1985.

[6] P. Erdős, A. Hajnal, E. C. Milner: Simple one-point extensions of tournaments, Mathematika 19 (1972), 57-62.

[7] R. Fraïssé, Sur certains relations qui généralisent l'ordre des nombres rationnels, C. R. Acad. Sci. Paris 237 (1953), 540-542.

[8] A. D. Gardiner, Homogeneous graphs, J. Combinatorial Theory (B) 20 (1976), 94-102.

[9] J. Hubička and J. Nešetřil, Finite presentation of homogeneous graphs, posets and Ramsey classes, preprint.

[10] A. H. Lachlan, Countable homogeneous tournaments, Trans. Amer. Math. Soc 284 (1984), 431-461.

[11] A. H. Lachlan and R. E. Woodrow, Countable ultrahomogeneous undirected graphs, Trans. Amer. Math. Soc. 262 (1980), 51-94.

[12] V. Müller, J. Nešetřil and J. Pelant, Either tournaments or algebras? Discrete Math, 11 (1975), 37-66.

[13] J. Nešetřil, Ramsey classes and homogeneous structures, Comb. Prob. Comput. (to appear). 
[14] R. Rado, Universal graphs and universal functions, Acta Arith. 9 (1964), 331-340.

[15] J. H. Schmerl, Countable homogeneous partially ordered sets, Algebra Universalis 9 (1979), 317-321. 